\title{
Functional Analysis of Finger Contact Locations during Grasping
}

\author{
Matei Ciocarlie* \\ Columbia University
}

\author{
Hao Dang ${ }^{\dagger}$ \\ Columbia University
}

\author{
Jamie Lukos $\ddagger$ \\ Arizona State University
}

\author{
Marco Santello $\$$ \\ Arizona State University
}

\author{
Peter Allen \\ Columbia University
}

\begin{abstract}
In this paper we present a method for studying human selection of fingertip contact point locations during grasping and manipulation. Our aim is to perform a functional analysis, looking at how a particular choice of contact point distribution affects the subjects' ability to resist external forces applied to the grasped object. We rely on grasp quality metrics derived from the Grasp Wrench Space, a method traditionally used for quantifying robotic grasps. We illustrate this approach by studying human grasps of a spherical object under a number of different disturbances. Our results indicate that the quality metrics we use can help explain the subjects' choice of contact points, although other possible grasping strategies may exist.
\end{abstract}

\section{INTRODUCTION}

Evolution has provided humans with incredibly sophisticated means of manipulation. The human hand, the most versatile endeffector known, is capable of a wide range of configurations and subtle adjustments. One important consequence is that, for a given grasping or manipulation task, there are multiple (or even an infinite number of) strategies that enable its successful completion. Examples of this range from redundant arm kinematics that allow multiple joint configurations to achieve a given hand location to complex actuation methods that enable multiple muscle activations to produce similar levels of fingertip forces.

In this paper we focus on the fingertip contact points between the human hand and a grasped object. The complexity of the human hand often enables it to achieve a huge number of possible contact configurations, particularly on objects of comparable scale. Before executing the task, humans must therefore select one configuration out of many candidates, and current research indicates that this choice is strongly influenced by the task itself [5].

Due to the kinematic and kinetic redundancy, many strategies are available to counteract potential disturbances to a grasp. At one extreme, subjects may use a force scaling strategy based on modulating individual fingertip forces as a function of expected disturbances. At the opposite extreme, subjects may use a contact point strategy where finger contact locations are chosen such as to minimize a given cost function (e.g., maximum force exerted by all digits required to counteract potential disturbances). Within these two extremes there exists a gradient of strategies. The choice of a specific strategy within this large range of choices is likely to be sensitive to the type of task (e.g., precision vs. force requirements) and individual differences among subjects (e.g., hand size and dexterity).

The choice of contact point locations during grasping and manipulation tasks can be of particular importance to the design of haptic devices. Consider first the case of tactile interfaces; while

\footnotetext{
*e-mail: cmatei@cs.columbia.edu

†e-mail: hd2181@columbia.edu

‡e-mail: jamie.lukos@asu.edu

§e-mail: marco.santello@asu.edu

『e-mail: allen@cs.columbia.edu
}

some implementations are highly portable and can be worn by the user directly on the finger (e.g. [12]), very often these devices have to be rigidly attached to the environment. As noted in the comprehensive review by Koudja et al. [1], electromagnetic actuation is one of the most commonly used methods, often leading to bulky and unportable packages. In the case of force feedback mechanisms, fully wearable solutions are also available, such as the Cybergrasp (Immersion Corp., CA). However, some of the most popular and widely used designs, such as the Delta and Omega (ForceDimension, Switzerland) and the Phantom (SensAble Technologies Inc., MA) allow the user to interface with the force-generating mechanism only by manipulating a tooltip, usually in the shape of a sphere or stylus.

While these approaches have significant practical advantages related to cost and manufacturing, they also impose constraints on where a user can place his fingers in order to interface with the haptic system. In order to understand the effect that such constraints have on the execution of grasping tasks, we propose to analyze how humans choose contact locations in an unconstrained environment. If we can understand how this choice is related to factors such as the geometry of the object, the task to be performed, or the expected disturbances, we can use these insights to design devices that enable more natural user behavior.

As part of this effort, this paper proposes a pipeline for analysing the contact points chosen by humans during grasping tasks. We perform this study from a functional perspective, looking not only at the contact locations in 3D Cartesian space, but also at the space of forces and torques that the resulting grasp can generate. Although related approaches are often used in the field of robotic grasp synthesis $(e . g$. $[3,14])$, to the best of our knowledge no applications for providing insights into the human grasping process have been proposed. We exemplify this method using a test case where users were required to grasp a spherical object in the presence of varying disturbances. We are also making the software components of our pipeline publicly available, meaning that other researchers can adapt it to suit any particular choice of target object and proposed tasks.

\section{FUNCTIONAL ANALYSIS OF CONTACT POINT LOCATIONS}

Multiple studies have underlined the human ability to adapt a grasp for a particular task by modulating the applied fingertip forces [9, 13, 15]; a review of this topic is also presented in [16]. This ability is important as it enables successful task completion even in the case where fingertip locations are constrained. However, it has also been shown that in the absence of such constraints humans choose different contact locations depending on the task to be performed $[5,8]$. Consider as an example the case where the task is to maintain a stable grasp of an object in the presence of an expected external disturbance: even though this task can be performed through contact force modulation, it was shown that subjects chose to also modulate the location of the contact points to the expected disturbance $[10,11]$.

One hypothesis for explaining this behavior is that a different choice of contacts can allow the grasp to resist the same disturbance by applying lower fingertip forces. However, no force sensors are currently available that allow for accurate recording of individual digits on a grasped object while still allowing subjects complete freedom to choose the positioning of their fingertip contact points. 
Therefore, in order to verify this hypothesis, we need to use a computational model to study the relationship between the force applied at each fingertip and the resulting wrench (combination of force and torque) applied by the grasp on the target object. The total space of wrenches that can be applied by the grasp is often referred to as the Grasp Wrench Space, or GWS. We note that the GWS and the space of disturbances that a grasp can resist are dual concepts, as in order to resist an external wrench, a grasp must apply an equal wrench in the opposite direction.

\subsection{Grasp Wrench Space construction}

As shown by Ferrari and Canny [7], the GWS can be constructed based on contact location information as well as object surface normals at the contact points. This approach requires knowing the space of wrenches that can be applied through each individual contact, also known as the Contact Wrench Space, or CWS. In this paper we use a CWS model that we have introduced in previous work [4]; for a given value of the contact normal force, this model predicts the maximum levels of tangential friction and frictional torque that the contact can also apply. Finally, each CWS is linearized and represented using a finite set of contact wrenches.

In general, a CWS (and implicitly a GWS) can be made arbitrarily large by simply increasing the level of normal force applied at each contact. In order to compute a "normalized" GWS, two options are presented in [7]. In both cases, the normal force applied at each contact is normalized to $1 \mathrm{~N}$. The first approach then builds the GWS as the convex hull of all individual CWS. In this case, the total force applied by the grasp is also normalized to $1 \mathrm{~N}$, and the GWS is considered to be of norm 1. The second approach is to build the GWS as the convex hull of the Minkowski sum of the contact wrenches that make up each CWS. In this case the total grasp force is bounded by the number of contacts, and the GWS is considered to have infinity norm.

$$
\begin{aligned}
\operatorname{GWS}_{L_{1}} & =\text { ConvexHull }\left(\bigcup_{\text {all contacts }} \mathrm{CWS}_{i}\right) \\
\mathrm{GWS}_{L_{\infty}} & =\text { ConvexHull }\left(\bigoplus_{\text {all contacts }} \mathrm{CWS}_{i}\right)
\end{aligned}
$$

The Minkowski sum operation allows us to consider the independent contributions of contacts on different fingers, but it quickly becomes intractable for large numbers of contacts. In this study we therefore use the $L_{1}$ GWS. We note however that it is also possible to build fast approximations of the $L_{\infty}$ GWS, particularly when only a portion of the space is of interest [2]. Although not reported here, we intend to further pursue this possibility in future work.

\subsection{Quality metrics}

The GWS is often used in the robotics literature to assess the quality of a grasp; in this study we use the same approach to analyze grasps executed by human subjects. A very common metric, often referred to as the Epsilon Quality of the grasp, or simply $Q_{\epsilon}$, is the radius of the largest ball that can fit inside the GWS. This is equal to the magnitude of the weakest disturbance that can break the grasp; therefore a high $Q_{\epsilon}$ indicates a grasp that is well suited for resisting any external wrench and is thus appropriate for use if the disturbances can not be known in advance.

In this study we are particularly interested in the subjects ability to adapt the grasp in the presence of known disturbances. Therefore, we also use a grasp quality metric that assesses its ability to resist a particular external wrench. Given a wrench $w$, this metric, which we refer to as $Q_{w}$, is equal to the scaling factor that can be applied to the GWS so that $w$ is on the surface of the GWS. This is equal to the minimum amount of total normal force that the fingers must apply in order for the grasp to resist the disturbance $w$.
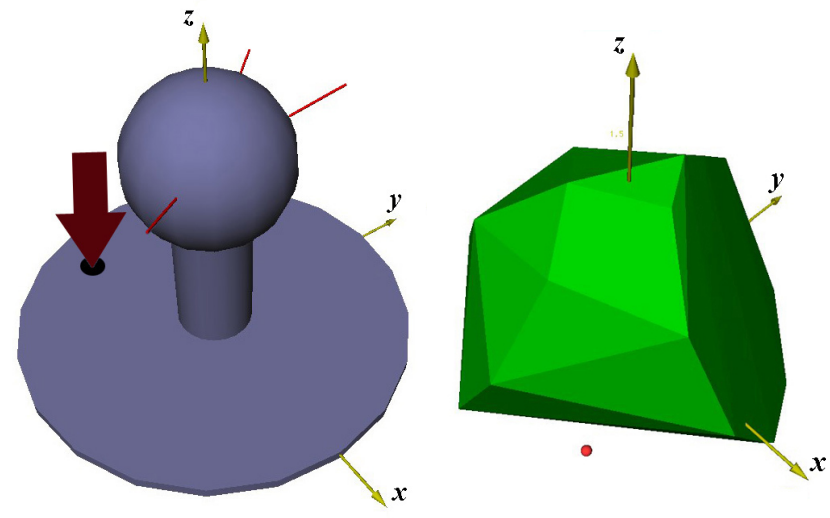

Figure 1: Grasp wrench space example. Left image shows target object and contact locations as thin red cylinders on the spherical top. Red arrow shows an external downwards force applied to a certain point on the object. Right image shows a 3-dimensional projection of the GWS using torques around the horizontal axes and force along the vertical axis. The projection of the external disturbance into the GWS is shown as a red marker.

We note that this is an "inverse" metric, in the sense that a smaller value of $Q_{w}$ indicates a grasp that is better suited at resisting the disturbance $w$ by applying low normal forces.

We also note that, in general, the GWS is 6-dimensional, encompassing combinations of 3-dimensional forces and 3-dimensional torques. While the quality metrics discussed above can be computed in an arbitrary dimensional space, a full 6-dimensional GWS is impossible to visualize. Furthermore, designing and executing tasks that test a grasp along all 6 dimensions of the GWS is a nontrivial exercise. Therefore, in this study we use 3-dimensional projections of the GWS, usually containing one dimension of force and two dimensions of torque. Further details on the particular choice of subspace as well as the experiments designed to use a 3-dimensional disturbance space are presented in the following section.

Figure 1 exemplifies these concepts. The left image shows an object along with the contact locations (the hand itself has been omitted for clarity). The right image shows a 3-dimensional projection of the GWS that contains the torques around the $x$ and $y$ axes and the force along the $z$ axis. The reference point for computing torque values is the origin of the coordinate system superimposed on the object. An external force oriented along the negative direction of the $z$ axis is applied to the object at the location shown in the image; the projection of this disturbance in the GWS is also shown as a red marker. Note that the downward vertical force also creates a positive torque around the $x$ axis and a negative torque around the $y$ axis.

In this section we have described the methods that we use for analyzing a grasp. This approach takes into account the geometry of the grasp, using normalized contact forces to build a GWS that is determined by the contact locations. These tools can be used to study whether a particular choice of contacts affords a grasp good overall quality, and also increased resistance against a particular disturbance. In the next section we apply them to a user study that investigates the subjects' choice of contact locations for a particular grasping task.

\section{MATERIALS AND METHOdS}

\subsection{Apparatus}

The goal of this experiment was to study the relationship between subjects' selection of contact points and a given wrench that the 

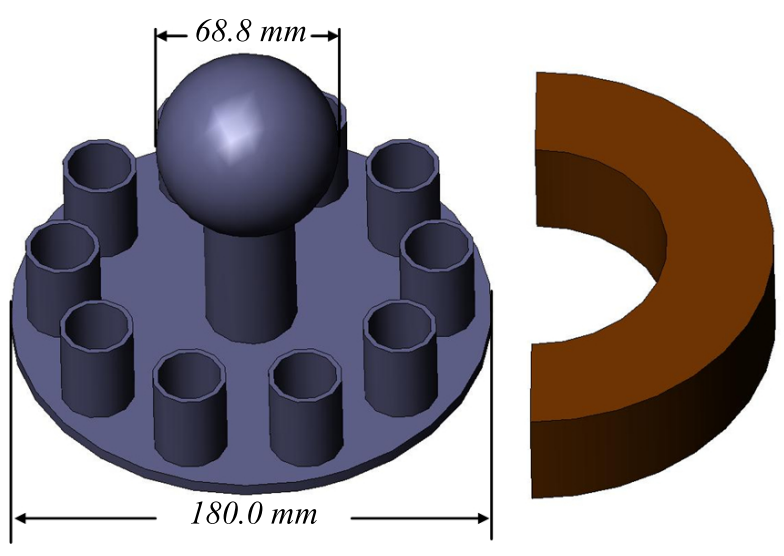

Figure 2: Target object used for human grasp recordings. The object consists of a spherical top that is the target of the grasp and a discshaped base with weight bins that are used to create disturbances. The cover part shown on the right can be used to conceal the weight bins (only one of two covers shown here).

grasp must resist. With this goal in mind we have designed the target object shown in Figure 2. This object consists of a spherical top attached to a disc-shaped base, with the sphere being the only part of the object that participants were allowed to grasp. Our choice of a spherical grasp area was based on two main reasons: first, it allows a complete choice of possible surface normals, allowing subjects to orient normal forces in any desired direction. Second, spherical tooltips are very common among force feedback devices, allowing for future studies that combine contact location and force information in behaviorally relevant settings.

The base of the object contains 10 bins, distributed equidistantly around its circumference. Any of these bins can hold a $100 \mathrm{~g}$ metallic weight. If a subject is asked to minimize object roll while grasping the object, an unbalanced set of weights placed in these bins can act as a disturbance that needs to be counteracted by applying appropriately distributed grasp forces. Considering the center of the base as a reference point, each weight creates a disturbance that is composed of a negative force along the $z$ axis, as well as torques around the $x$ and $y$ axes. By varying the number and location of the weights we can effectively sample this 3 -dimensional wrench space and create different disturbances.

Finally, it is also possible to use the two additional parts, such as the one shown in Figure 2 on the right, to cover the weight bins. This prevents the subject from anticipating the disturbance based on vision, without affecting access to the graspable part of the object. All the experiments discussed in this paper were performed with the weight bins concealed. However, this design can also enable more complex future experiments that compare the effects of different stimuli, such as visual perception of the disturbance during pregrasp and haptic perception during the grasp itself.

\subsection{Experimental procedure}

Six subjects gave informed consent to participate in this study as approved by the Institutional Review Board at Arizona State University and were in accordance with the Declaration of Helsinki. Subjects sat at a table on which the object shown in Figure 2 was placed approximately $25 \mathrm{~cm}$ in front of them, aligned with their midline. We asked subjects to reach, grasp, lift and replace the object using a tripod grasp (thumb, index, and middle fingers). While subjects were allowed complete freedom in choosing contact point locations, the choice of enforcing a tripod grasp was made in order to increase uniformity between trials. Our method assumes each

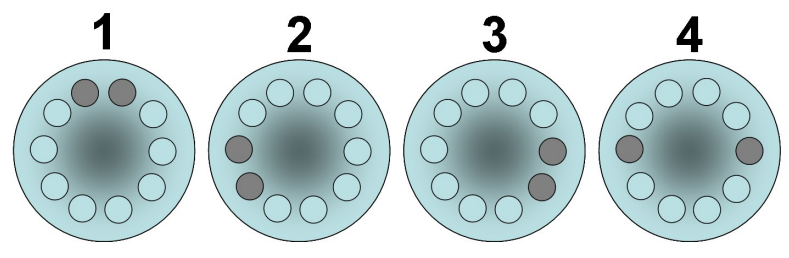

Figure 3: Disturbances created by placing 2 weights in the bins of the test object, using 4 different configurations.

\begin{tabular}{|c|cccccc|}
\hline $\begin{array}{c}\text { Subject } \\
\text { Avg. standard } \\
\text { deviation (mm) }\end{array}$ & 14.96 & 7.87 & 15.98 & 19.00 & 11.60 & 7.53 \\
\hline
\end{tabular}

Table 1: Modulation of fingertip position. For each subject, the standard deviation of fingertip contact location over the 40 blocked trials is shown, averaged over all three fingers used in the grasp.

contact has identical force-generating capability, therefore changing the number of contacts can affect the computation of the quality metric. However, this approach might also reduce the range of grasp strategies used. Follow-up studies will quantify this aspect by recording the number of fingers used spontaneously, in the absence of such restrictions, and comparing the results to those from enforced tripod grasps.

In order to add an external disturbance to each grasp, we placed 2 weights in the bins of the object using 4 different configurations. Each of these configurations, shown in Figure 3, created a different combination of forces and torques (with disturbance 4 being the only one with a zero net torque). Subjects were not shown where the weights were placed or told how/where to grasp the sphere, but only to lift it as vertically as possible approximately $10 \mathrm{~cm}$ off the table. These instructions allowed subjects to voluntarily choose fingertip contact points and force distributions on the object.

Grasps using each of the four disturbances were tested in two conditions: 1) blocked, where the same disturbance was used for 10 consecutive trials, and 2) random, where the disturbance was changed on a trial-to-trial basis. Each participant executed a total of 40 trials in each of the two conditions (i.e. 80 trials total). The blocked condition allowed subjects to anticipate the disturbance after the initial trial, thus allowing for planned digit placement and forces on the object. Alternatively, the random condition prevented subjects from using anticipatory control. In the rest of this study we will distinguish grasps performed in these two conditions by referring to them as "blocked grasps" and "random grasps" respectively.

The motion of the hand during reach-to-grasp was determined by tracking infrared light-emitting diodes placed on the fingernails of each digit using the Optotrak Certus motion tracking system (100 $\mathrm{Hz}$; Northern Digital Inc., Waterloo, ON, Canada). After collection, data were run through a $6 \mathrm{~Hz}$ low-pass Butterworth filter. Digit contact time was determined as the time at which the tangential velocity of each fingertip reached its minimum value prior to object lift onset. The locations of the contact points on the object were obtained by projecting the recorded fingertip marker locations on the surface of the sphere. Friction between human skin and the thermoplastic material of the target object was modeled using a static coefficient $\mu=0.5$ [6]. This data were then used to build the GWS and compute various grasp quality metrics, as discussed in the previous section.

\section{Results}

The first aspect of the data that we analyzed was whether the subjects chose different contact locations as the external disturbance 

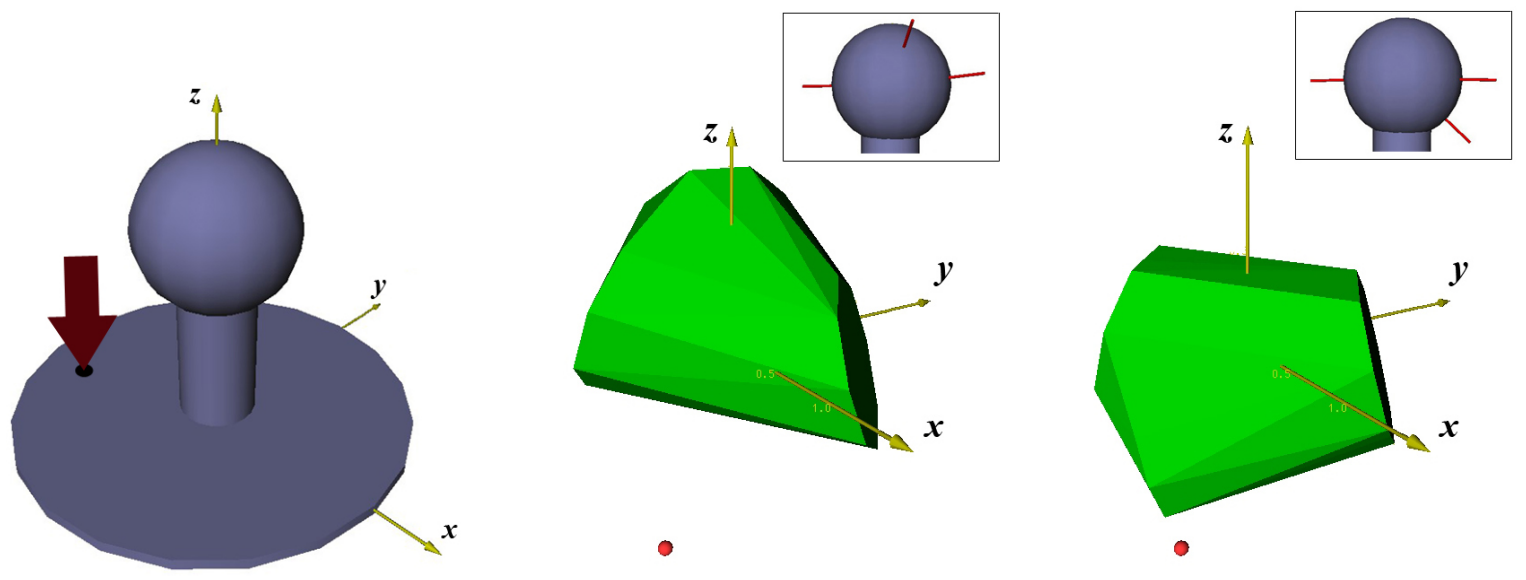

Figure 4: The Grasp Wrench Space can show the adaptation of a human grasp to resist a particular disturbance. Left: target object and applied disturbance; Middle: GWS of a grasp from random trial; Right: GWS of a grasp from blocked trial. In both GWS images, the red marker shows the projection of the disturbance.

\begin{tabular}{|cc|cccc|}
\hline \multicolumn{2}{|c|}{ Disturbance $w$} & 1 & 2 & 3 & 4 \\
\hline \multirow{2}{*}{ AD and MA } & $Q_{w}$ random & 2.43 & 2.37 & 2.22 & 2.11 \\
& $Q_{w}$ blocked & 1.91 & 2.01 & 1.71 & 1.69 \\
\hline \multirow{2}{*}{ Other 4 } & $Q_{w}$ random & 1.88 & 1.64 & 1.75 & 1.64 \\
& $Q_{w}$ blocked & 1.97 & 1.71 & 1.80 & 1.82 \\
\hline
\end{tabular}

Table 2: Comparison between random and blocked trials. For each disturbance $w$ we compare the $Q_{w}$ metric of the grasps in the random trials against those in the blocked trials using $w$.

wrench was varied across the trials. Table 1 shows the standard deviation of fingertip positions at contact over the complete set of blocked trials, for each of the six subjects. We note that this is strictly a measure of the variance in the geometry of the contacts, without any force calculations involved. Our findings showed that all subjects varied their choice of contact locations, but to different extents, ranging from $7 \mathrm{~mm}$ to $19 \mathrm{~mm}$ for each finger.

Having confirmed the presence of geometric variance in the data, we performed the functional analysis described in Section 2. This approach enabled us to investigate whether the choice of different contact locations across trials allowed the subjects to better resist known disturbances, according to our quality metrics. For each recorded grasp we built a 3-dimensional GWS as described in the previous section; the GWS was used to compute the value of the $Q_{w}$ quality metric for each disturbance $w \in\{1,2,3,4\}$. We then compared the results obtained in the random trials (when subjects did not know what disturbance to expect) against those from blocked trials (which allowed subjects to anticipate the disturbance).

The results are presented in Table 2. They show that two of the subjects (AD and MA) consistently chose grasps that afforded better disturbance resistance in the case where the disturbance was expected. This behavior was observed over the full set of possible disturbances. However, the results from the other 4 subjects did not display the same trend, and showed no adaptation of the grasp to the expected disturbance, according to the used quality metric. The different behavior between these two groups of subjects could potentially be attributed to a number of different factors; we will expand on this topic in the next section.

An interesting note is that even in the case when subjects adapted to a particular known disturbance, this did not affect the overall ability of the grasp to resist unknown wrenches. This ability, measured

\begin{tabular}{|c|c|c|c|c|c|}
\hline \multicolumn{2}{|c|}{ Disturbance $w$} & 1 & 2 & 3 & 4 \\
\hline \multirow{2}{*}{$\begin{array}{l}\mathrm{AD}, \mathrm{MA} \\
\text { and JW }\end{array}$} & $\begin{array}{c}Q_{w}-\text { first trial } \\
\text { in block }\end{array}$ & 3.26 & 2.50 & 2.82 & 1.94 \\
\hline & $\begin{array}{c}Q_{w}-\text { avg. of other } \\
\text { trials in block }\end{array}$ & 1.91 & 1.94 & 1.70 & 1.88 \\
\hline \multirow{2}{*}{$\begin{array}{l}\text { WH, OF } \\
\text { and SI }\end{array}$} & $\begin{array}{c}Q_{w} \text { - first trial } \\
\text { in block }\end{array}$ & 1.79 & 1.58 & 1.98 & 1.55 \\
\hline & $\begin{array}{c}Q_{w}-\text { avg. of other } \\
\text { trials in block }\end{array}$ & 1.91 & 1.68 & 1.83 & 1.67 \\
\hline
\end{tabular}

Table 3: Adjustment to a know disturbance in blocked trials. For each disturbance $w$ we compare the $Q_{w}$ metric of the grasp for the first trial of the block against its average for the other trials in the same block.

using the $Q_{\epsilon}$ metric, showed no significant variance between the random and blocked conditions for any of the six subjects, with an average value in each case of 0.34 .

One advantage of using a 3-dimensional GWS is that it can be visualized directly. Figure 4 illustrates this using two trials from the subject $\mathrm{AD}$, and comparing the ability of the grasp to resist a particular disturbance. One grasp is from the random condition, where the subject was unaware of the applied disturbance. The other grasp is taken from a block of trials with the same disturbance applied; its adaptation to resist it can be directly observed through visual inspection of the GWS. This can also be quantified using the $Q_{w}$ metric: in this case, it has a value of 3.20 for the random grasp and 1.56 for the blocked grasp, showing that the latter needs to apply less than half the force of the former in order to resist the given disturbance. However, visual inspection can also reveal which other regions of the GWS (if any) have suffered due to this adaptation. Not surprisingly, in the case shown here the blocked grasp is ill-equipped to resist wrenches in the opposite direction of the disturbance that is is adapted for.

Finally, a direct measure of adaptation to a disturbance is the change between the first trial in a block (where the subject does not yet know which disturbance to expect) and the subsequent trials in the same block, executed using the same disturbance. As shown in Table 3, the results again divided our subjects into two groups, with half of the subjects showing that after the execution of the first trial in a block, the grasp was adapted to allow better resistance to the disturbance. 


\section{Discussion AND CONCLUSIONS}

The results presented here show that, when required to grasp a spherical object and resist an external disturbance consisting of a combination of force and torque, participants chose different contact locations across different trials. In this paper we have presented an approach that uses the Grasp Wrench Space to assess whether the choice of contact locations enables a grasp to better resist a disturbance while applying less total force. Using collected human grasp data, we observed two trends amongst the subjects of our study. When faced with a known disturbance, one group of participants chose contact locations that were well adapted to resist it, according to the used quality metrics. The other group showed no such correlation between the GWS and the expected wrench.

It is important to note that even if, on a given trial, a subject chose contact point locations affording better disturbance resistance, this does not necessarily imply that the subject took advantage of this by applying smaller forces. Furthermore, the interpretation of the difference in modulation of contact points can also reflect betweensubject differences in their ability to associate/understand optimal contact point location. In other words, the fact that some subjects modulated contact points significantly does not necessarily imply that this modulation was optimal under the strategy discussed here. The discriminating factor is the adeptness of the subject at performing the required task, reflected in the ability to minimize object tilt during the grasp. In future work we plan to include this measurement in our analysis.

As discussed in the introduction, the nature of the human grasping mechanism allows for multiple strategies for performing this task; consequently, these results are not unexpected. The analysis method described here is equipped to investigate one potential strategy, related to the GWS; other possibilities include modulation of fingertip force, wrist impedance, etc. Our results show that it is necessary to design experiments that can better discriminate between such strategies. One possible approach would be to measure all contact forces without restricting the choice of contact locations; however, an experimental setup that would enable such studies does not exist yet. Other possibilities include the application of larger magnitude disturbances that would discourage adaptation solely through force modulation, larger object surface to encourage more possible contact locations and also tasks with greater behavioral consequences or relevance to the subject, such as avoiding spilling water from a glass.

In this study, we showed that human choice of contact locations during grasping and manipulation can partly be explained by understanding the nature of the task and the expected disturbances. The software tools that we used are being made publicly available to the research community, as they can also be applied to different task spaces or objects, specific to a particular mechanism design. We believe that this approach can help the design of better haptic devices and interfaces, and in the process enable new insights into human grasping and manipulation.

\section{ACKNOWLEDGEMENTS}

The authors would like to thank Lisa Bobich and Qiushi Fu for helping with data collections. This work was funded in part by NIH BRP grant 1RO1 NS 050256-01A2 and NSF Grant BCS 0819547.

\section{A SOFTWARE AVAILABILITY}

All the GWS analysis discussed in this paper, including computation of the quality metrics, were performed using the GraspIt! simulator developed in the Robotics Lab at Columbia University. GraspIt! is available for download at:

http://www.cs.columbia.edu/ cmatei/graspit

\section{REFERENCES}

[1] M. Benali-Khoudja, M. Hafez, J. Alexandre, and A. Kheddar. Tactile interfaces: a state-of-the-art survey. In International Symposium on Robotics, Paris, France, March 2004.

[2] C. Borst, M. Fischer, and G. Hirzinger. A fast and robust grasp planner for arbitrary 3D objects. In IEEE International Conference on Robotics and Automation, pages 1890-1896, Detroit, MI, May 1999.

[3] C. Borst, M. Fischer, and G. Hirzinger. Grasp planning : How to choose a suitable task wrench space. In IEEE International Conference on Robotics and Automation, New Orleans, LA, April 2004.

[4] M. Ciocarlie, C. Lackner, and P. Allen. Soft finger model with adaptive contact geometry for grasping and manipulation tasks. In Joint Eurohaptics Conf. and IEEE Symp. on Haptic Interfaces, 2007.

[5] R. Cohen and D. Rosenbaum. Where grasps are made reveals how grasps are planned: generation and recall of motor plans. Experimental Brain Research, 157(4):486-495, 2004.

[6] S. Comaish and E. Bottoms. The skin and friction: deviations from Amonton's laws, and the effects of hydration and lubrication. British Journal of Dermatology, 84:37-43, 1971.

[7] C. Ferrari and J. Canny. Planning optimal grasps. In IEEE Intl. Conf. on Robotics and Automation, pages 2290-2295, 1992.

[8] J. Friedman and T. Flash. Task-dependent selection of grasp kinematics and stiffness in human object manipulation. Cortex, 43:444-460, 2007.

[9] F. Gao, M. Latash, and V. Zatsiorsky. Maintaining rotational equilibrium during object manipulation: linear behavior of a highly nonlinear system. Experimental Brain Research, 169:519-531, 2006.

[10] J. Lukos, C. Ansuini, and M. Santello. Choice of contact points during multidigit grasping: Effect of predictability of object center of mass location. Journal of Neuroscience, 27(14):3894-3903, 2007.

[11] J. Lukos, C. Ansuini, and M. Santello. Anticipatory control of grasping: independence of sensorimotor memories for kinematics and kinetics. Journal of Neuroscience, 28(48):12765-12774, 2008.

[12] K. Minamizawa, H. Kajimoto, N. Kawakami, and S. Tachi. A wearable haptic display to present the gravity sensation - preliminary observations and device design. In Joint EuroHaptics Conference and Symposium on Haptic Interfaces for Virtual Environment and Teleoperator Systems, pages 133-138, Tsukuba, Japan, 2007.

[13] R. Reilmann, A. M. Gordon, and H. Henningsen. Initiation and development of fingertip forces during whole-hand grasping. Experimental Brain Research, 140:443-452, 2001.

[14] M. Roa and R. Suarez. Geometrical approach for grasp synthesis on discretized $3 \mathrm{~d}$ objects. In IEEE-RSJ Intl. Conf. on Intelligent Robots and Systems, 2007.

[15] M. Santello and J. Soechting. Force synergies for multifingered grasping. Experimental Brain Research, 133(4):457-467, 2000.

[16] V. Zatsiorsky and M. Latash. Multifinger prehension: An overview. Journal of Motor Behavior, 40:446-475, 2008. 